\title{
Exploring Co-Parent Experiences of Sexuality in the First 3 Months after Birth
}

\author{
Sari M. van Anders, $\mathrm{PhD},{ }^{*}$ Lauren E. Hipp, $\mathrm{BS},{ }^{\dagger}$ and Lisa Kane Low, $\mathrm{PhD}^{\ddagger}$ \\ *Departments of Psychology \& Women's Studies, Program in Neuroscience, Reproductive Sciences Program, University \\ of Michigan, Ann Arbor, MI, USA; 'Department of Psychology, University of Michigan, Ann Arbor, MI, USA; ${ }^{\ddagger}$ School of \\ Nursing, Department of Women's Studies, University of Michigan, Ann Arbor, MI, USA
}

DOI: $10.1111 / \mathrm{jsm} .12194$

\section{A B S T R A C T}

Introduction. Research on postpartum sexuality has focused primarily on mothers, though new findings suggest that relational perceptions may have a strong influence over sexual desire and behavior. Little investigation exists regarding sexuality in partners of postpartum women. Additionally, recent findings point to the importance of a partner's sexuality for postpartum women's perceptions of their own sexuality in this time.

Aims. The goal of this research was to explore women's partners' sexuality in the early postpartum phase taking into account psychosocial context.

Methods. Partners ( $\mathrm{N}=114$; 95 men, 18 women, 1 unspecified) of postpartum women completed a retrospective online questionnaire about their sexuality during the 3 months following their youngest child's birth.

Main Outcome Measures. Primary measures included sexual desire (Sexual Desire Inventory), latency to sexual behavior, and enjoyment and initiation of sexual behavior. Other psychosocial variables were investigated: partners' perceptions of the birth mother's sexual desire, perceptions of the birth experience (Questionnaire Measuring Attitudes About Labor and Delivery), postpartum stress (Perceived Stress Scale), body image self-consciousness (Body Image Self-Consciousness Scale), social support (Multidimensional Scale of Perceived Social Support), fatigue, and experiences surrounding breastfeeding.

Results. Partners reported most frequent engagement in intercourse in the postpartum period, earliest engagement in masturbation, and highest enjoyment of receiving oral sex compared with other sexual activities. Partners' sexual desire was not correlated with the psychosocial variables measured in the study. Findings for partners' sexuality were similar by gender, except for perceptions of social support and likelihood to engage in intercourse.

Conclusions. This study provided a novel perspective on the study of postpartum sexuality by investigating physical and psychosocial influences on the experiences of partners of parous women. Given parallels between sexuality reported by partners in this study and by birth mothers in past studies, this study provided evidence that sexuality in the postpartum period may be experienced similarly, highlighting the social and relational nature of the postpartum. van Anders SM, Hipp LE, and Kane Low L. Exploring co-parent experiences of sexuality in the first 3 months after birth. J Sex Med 2013;10:1988-1999.

Key Words. Postpartum; Sexuality; Partner; Co-Parent; Birth; Desire; Sexual Behavior

\section{Introduction}

$\mathrm{T}$ he transition to parenthood involves physical, social, relational, and sexual adjustments, and-though the majority of postpartum studies attend to new mothers-this is true for the partners, or co-parents, of new mothers as well. Current research brings the partner (which includes husbands and same- or other-sex partners of parous women who are sharing in parenting [i.e., co-parents]) into focus by showing that birth mothers' perceptions of their partner's postpartum sexuality is associated with mothers' reported sexual experiences after birth [1]. Mothers who perceived their partners' desire as high during the postpartum period scored higher on their own measures of sexual desire, and the reported importance of fulfilling a partner's desires statistically contributed to earlier performance of oral sex on partners compared with latency to engage in other 
activities such as masturbation, intercourse, and receptive oral sex [1]. Furthermore, other studies have found that women's sexual activity is often motivated in part by attention to their partner's sexual satisfaction [2]. This relational sexuality, however, is not limited to women. Past research has shown that male partners reported changes in their wives' sexual responsiveness as the only negatively changing aspect of their marital relationship [3], and decreased desire from the birth mother was cited as the most common reason for negative postpartum sexual adjustment [4]. These limited findings are suggestive that postpartum sexuality merits study, not only in birth mothers but also their partners. ${ }^{1}$

\section{General Trends in Partner Sexuality}

Relatively few studies have looked at sexuality in partners during the postpartum phase, and extant research has focused entirely on male partners. These studies show a consistency in men's levels of sexual desire at varying time points across the transition to parenthood $[5,6]$. Despite these stable rates of sexual interest throughout the postpartum period, multiple studies note a decrease in sexual frequency within the first year after birth $[5,6]$. Moreover, declines in sexual satisfaction were observed in men even when there were no significant changes in sexual frequency across the first-year postpartum [7]. Together, these findings suggest that perceptions of postpartum sexuality may be related to psychosocial factors more than sexual engagement itself. These discrepancies between desire, satisfaction, and sexual frequency may contribute to a shift ${ }^{2}$ in sexual behavior toward noncoital partnered activity [8] or masturbation $[9,10]$, and high reports of men's vs. women's initiation of sexual behavior in the postpartum period [2]. Despite these adjustments, many men report acceptance of abstinence after the birth, noting that it was necessary for their partner to heal and for the overall well being of their new family [11]. These findings suggest a complexity in partners' sexual experiences, with adjustments in behavior and changes to satisfaction that may or may not be in line with each other.

\footnotetext{
${ }^{1}$ And also, of course, adoptive parents, who were outside the purview of the current research study.

${ }^{2}$ And we do not mean to suggest that this shift, including more masturbation, noncoital activity, or male initiation is de facto problematic or undesirable.
}

\section{Relational Influences}

Though multiple studies have shown that relationship satisfaction significantly deteriorates from 6 months to 1 year postpartum and beyond in men, few studies have explored the factors that might instigate this pattern $[7,12]$. Investigations into the dyadic adjustment of postpartum couples suggest that feelings of jealousy or exclusion from the mother-infant relationship may contribute to these patterns $[11,13,14]$, especially if the mother is breastfeeding or if the father has taken on the role of financial provider for the family, leaving him fewer opportunities to become involved in the daily intimacies of childcare [15]. Accordingly, relationship contexts and perceptions may be important to understanding partners' sexuality during the postpartum period.

\section{Birth-Related Influences}

Though factors related to birth experience and vaginal injury have been extensively studied in women, their partners' experiences have been largely excluded from this discussion, perhaps due to understandings of birth and pregnancy that are located in women's bodies as opposed to conceptualizing them as social or relational events. Only two studies have investigated the effect of birth experience on sexuality in women's partners and they focused on how birth delivery method might influence male sexual dysfunction. Gungor et al.'s examination of male sexual dysfunction found no significant differences in reports of sexual pleasure between men with partners who were nulliparous, delivered by elective Cesarean (C) sections, or delivered vaginally with episiotomy [16]. However, another study found higher sexual dysfunction in men whose partners had spontaneous vaginal deliveries, assisted vaginal deliveries, or emergency $\mathrm{C}$ sections compared with those who had planned $\mathrm{C}$ sections [17]. Unfortunately, studies that focus on dysfunction preclude the study of birth experience as a positive factor for partner sexuality, and no studies have focused solely on the association between mode of delivery and sexual enjoyment or sexual desire. Several studies have shown that husbands fear breaking stitches or hurting their wives, feelings that may lead to decreased sexual frequency $[2,18]$. These findings thus suggest that the perceptions of the delivery experience and assumptions of lasting physical effects may also have important impacts on male sexuality in the postpartum period. 


\section{Breastfeeding Influences}

Much of the discourse surrounding the influence of breastfeeding on postpartum sexuality centers around the hormonal changes in lactating women. However, research has also shown that women's choices to breastfeed can influence how their partners view their relationship and sexuality. Studies suggest that breastfeeding can provoke conflicting feelings in male partners as they knew it was good for the baby but often feel left out of the motherinfant dyad during feeding times $[15,19]$. Influences of breastfeeding on sexuality are also not clearly negative, as $59.5 \%$ of men in one study responded that breast changes in the birth mother during the postpartum period had no effect on the men's sexuality [18], though other studies note that men avoid their partners' breasts at this time due to the belief that they are exclusively for the baby [11]. These contradictory findings suggest that breastfeeding's effects on sexuality in men can be multifaceted and moderated by their perceptions of the breastfeeding process. To our knowledge, there are no studies that investigate breastfeeding-related experiences of same-sex partners.

\section{Existing Limitations}

As has been noted, a critical limitation of research on partners' experiences of sexuality during the postpartum period has been the exclusive focus on men, primarily husbands, effectively ignoring the experiences of lesbian partners and unmarried couples. The studies to date have contained small sample sizes, making broader patterns less visible. Moreover, research questions tend to be grounded in the assumption that birth and new parenthood impinge negatively on male partners' sexuality even to a clinical degree because of biomedical processes related to birth and breastfeeding as opposed to contextual and relational factors like relational changes, fatigue, and social support, etc. Conceptualizations of sexuality following childbirth have rarely included positive attributes. Lastly, the few studies that do include women's partners are limited by a focus on comparing mothers and partners. This approach seems grounded in the arguably untested assumption that mothers' and partners' sexualities are of the same kind. The present study aims to examine partners' experiences of sexuality in the postpartum phase, including a broad spectrum of contextual, relational, and social factors.
As this paper attempts to address the above limitations while using an inclusive definition of women's partners sensitive to gender, sexual, and relational diversity, it is important to also attend to terminology limitations when discussing this population. There currently is no universal term proposed for studying the partners of parous women. Previous studies have discussed this population using terms of husbands or fathers. The use of the term "husband" privileges marital relationships over others, assumes that partners are male, and also foregrounds the adult relationship potentially pushing the primary parenting role again onto mothers. The term 'fathers', on the other hand, assumes that co-parents are male and foregrounds the parental relationship over the partner relationship. The general use of the term "partner," though more inclusive, also backgrounds the relationship between the partner and the new child by focusing solely on his or her connection to the birth mother and does not specify that the partner is also a parent. Similarly, the term "co-parent" foregrounds the parenting association and backgrounds-or even erases - the adult relationship. Still, a term must be used, and we will accordingly use co-parent throughout to refer to individuals involved in both romantic/sexual relationships with birth mothers and parenting relationships with the newborn. Though the term "co-parent" is commonly thought to include both members of a parenting relationship, the use of the term here will not extend to birth mothers.

\section{Aims}

This research aims to explore the physical, social, relational, and psychological experiences of co-parents during the first 3 months after their child's birth to determine how these experiences influence their experience of sexuality. This study focuses specifically on sexual behavior and sexual desire and aims to incorporate the experiences of diverse co-parents, including women in samesex partnerships, to expand our understanding of co-parents' experiences beyond the current heterosexual focus.

\section{Methods}

\section{Participants}

Co-parents $(\mathrm{N}=114)$ over the age of 18 years old who have had a child in the past 7 years were recruited to participate in this study (Table 1). We 
Table 1 Sample demographics

\begin{tabular}{lc}
\hline Demographics & $\mathrm{N}(\%)$ \\
\hline Age & 114 \\
$<20$ & $4(3.5)$ \\
$20-24$ & $28(24.5)$ \\
$25-29$ & $30(26)$ \\
$30-34$ & $24(21)$ \\
$35-39$ & $17(15)$ \\
$40+$ & $11(10)$ \\
Age at most recent birth & 114 \\
$<20$ & $15(13)$ \\
$20-24$ & $32(28)$ \\
$25-29$ & $31(27)$ \\
$30-34$ & $17(15)$ \\
$35-39$ & $14(12)$ \\
$40+$ & $5(4)$ \\
Birth type & 114 \\
Single & $112(98)$ \\
Multiple & $2(2)$ \\
Delivery method & 113 \\
Vaginal & $81(72)$ \\
Assisted vaginal & $4(3.5)$ \\
Planned cesarean & $13(11.5)$ \\
Emergency cesarean & $15(13)$ \\
Sexual orientation/identity & 114 \\
Heterosexual & $89(78)$ \\
Gay/lesbian & $15(13)$ \\
Bisexual & $4(4)$ \\
Other & $6(5)$ \\
Postpartum relationship status & 113 \\
Dating & $6(5)$ \\
Committed with one partner & $102(90)$ \\
Separated and co-parenting & $1(1)$ \\
Multiple commited relationships & $4(4)$ \\
\hline & \\
&
\end{tabular}

selected this time frame because it maximized the likelihood of recall balanced against the need to recruit broadly enough to complete the study. Participants had to have been involved in parenting that child during the 3 -month period following the child's birth and also had to have been involved in a sexual relationship during that time. Recruitment was carried out through online advertisements, community postings, and Amazon's Mechanical Turk (MTurk) [20], including targeted ads toward women in same-sex relationships. Participants who had experienced intimate partner violence during the postpartum period were provided with information on survivor help lines and were exited from the questionnaire. Participants included in the analyses varied in educational background, occupation, and income level. Co-parents ranged between 19 and 54 years of age, with a mean age of 29 (standard deviation $[S D]=7.241)$. Participants' ages at the time of their most recent birth experience ranged from 14 to 48 years old (mean age $=27, \mathrm{SD}=6.813$ ), while the average time since their most recent birth was 2.46 years $(\mathrm{SD}=1.786)$.

\section{Procedure}

Approval for this study was obtained from the University of Michigan Institutional Review Board. Recruitment advertisements directed participants to an online screening questionnaire to determine their eligibility. Eligible participants then consented to participate and proceeded to an online survey that consisted of a Health and Background Questionnaire to assess population demographics, as well as sexual orientation/identity and relationship status during the first 3 months after the birth of their last child. Participants were also asked to respond to a series of questions regarding their experiences surrounding their partner's (i.e., the birth mother's) most recent pregnancy and their own birth and postpartum experiences, defining the postpartum period as the 3 months after the birth of the participant's youngest child. Participants were then compensated through entry into a raffle to win one of the two $\$ 50.00$ prizes, or if they had completed the questionnaire through Amazon's MTurk, they were compensated with $\$ 0.30$ per assignment (which is how MTurk defines tasks like research participation). Data collection for this study a separate study conducted in parallel with a previously published study on birth mothers [1]; however, questionnaire items and standardized scales were reworded to reflect the experiences of co-parents in contrast to the previously analyzed experiences of birth mothers. To be clear, the co-parents in this investigation were not necessarily co-parents of the mothers in our previous study [1]. Analyses for these two studies were conducted separately by birth mother vs. co-parent as planned because of our interest in understanding postpartum sexuality in a localized context.

\section{Materials}

All instruments used in this investigation are previously described in Hipp et al. [1], though questionnaire items were rephrased to target co-parents for use in this study. All standardized questionnaires were reworded to reflect feelings during the 3-month period following their youngest child's birth for retrospective analysis.

\section{Birth Experience Items}

Participants' experiences surrounding the birth of their youngest child were measured using the Questionnaire Measuring Attitudes on Labor and Delivery (QMAALD [21]), where items were rephrased to assess co-parents' feelings during their partner's labor and delivery (e.g., How scared 
were you during your partner's delivery? How confident were you during your partner's labor and delivery?). Higher scores indicated more positive feeling surrounding the birth. Additionally, co-parents were asked to rate how they felt their partner's experiences surrounding the birth affected her sexual desire toward them on a sevenpoint scale where 1 indicated a negative impact on desire and 7 a positive influence. Due to the fact that the researchers were interested in how the birth experience might influence co-parents' experiences of sexuality, the participants were also asked to indicate their partner's delivery method and whether or not their partner experienced specific birth interventions.

\section{Postpartum Experience Items}

Due to the exploratory nature of this study, multiple psychosocial variables were investigated including perceptions of social support via the Multidimensional Scale of Perceived Social Support (MSPSS) [22], perceptions of postpartum stress using the Perceived Stress Scale (PSS) [23], and co-parents' body image self-consciousness using the Body Image Self-Consciousness Scale (BISC) [24]. Additionally, co-parents were asked to indicate how their baby was fed during the postpartum period and to rate their own average energy level during that time on a seven-point scale, with 1 indicating "extreme fatigue" and 7 indicating "high energy."

To investigate opinions on traditional gender roles in co-parents, participants were asked to rate their agreement to a series of five stereotypical gender role statements. Participants were presented with statements such as "A woman cannot give her children the attention they need if she works outside the home" and "Financial responsibility in a family should reside on the father or someone other than the mother." Participants then indicated their agreement on a scale where 1 indicated "very strongly disagree" while 7 indicated "very strongly agree." Scores on these five statements were summed such that higher scores indicated higher agreement with traditional gender roles.

\section{Sexuality-Related Items}

Co-parents were asked to indicate which sexual activities they engaged in before birth (e.g., sexual intercourse, oral sex, and masturbation), as well as which activities they had resumed during the first 3 months after the birth of their youngest child. Intercourse was defined for participants as any sexual activity involving penetration. Co-parents who had engaged in sexual activity during the postpartum phase were asked to indicate their enjoyment of each individual activity as well as whether they or their partner had initiated the activity first in the postpartum period.

Measures of co-parents' sexual desire during the postpartum period were obtained by the use of the Sexual Desire Inventory (SDI) [25]. An additional question was included to ask the participant to compare their level of sexual desire with that of their partners. To further investigate the influences on sexual desire in this population, co-parents were given a set list of 15 factors compiled from previous literature for their associations with changes in maternal postpartum sexuality and from limited studies on co-parent sexuality. Participants were then asked to select three factors and order them as first, second, and third most contributing to their bighest experience of sexual desire within the first 3 months after their child was born. Participants were then presented with the same list and asked to follow the same procedure, this time indicating the factors that contributed most to their lowest experience of postpartum sexual desire. Participants were also able to type in and rank their own provided factor if that factor was not included in the set list.

As a goal of this study was to investigate how perceptions of a partner's sexuality influenced the sexual desire and sexual behavior of co-parents of parous women, participants also answered three questions taken from the SDI [25] but reworded to assess their partner's postpartum sexuality. These three questions were then summed to obtain a partner perception score such that higher scores indicated co-parents' perceptions of their partner's (i.e., the birth mother's) sexual desire as high following childbirth. In a separate item, co-parents indicated how important it was for them to fulfill their partner's sexual desires on an eight-point scale where 1 was "not at all" and 8 was "extremely important."

\section{Main Outcome Measures}

The main outcome measures presented in this study were those specific to co-parents' experiences of sexuality in the postpartum period, including resumption of sexual behavior, retrospective reports of personal sexual desire via the SDI [25], perceptions of a partner's sexual desire after childbirth, and identification of factors that 
influenced sexual desire in the postpartum period. Other outcome measures targeted psychosocial factors including perceptions of the birth experience (QMAALD), body image self-consciousness (BISC) [24], postpartum stress (PSS) [23], perceptions of social support (MSPSS) [22], how their baby was fed, and personal energy level on a typical day postpartum.

\section{Results}

An exploratory approach was employed to investigate co-parents' physical, social, psychological, and relational experiences during the 3-month postpartum period, emphasizing how these factors influenced sexual behavior and desire during this time. Data were analyzed using the Statistical Package for the Social Sciences (SPSS Inc., Chicago, IL, USA).

\section{Trends in Co-Parents' Postpartum Sexual Behavior}

Co-parents' engagement in sexual activity (e.g., intercourse, masturbation, and oral sex) during the postpartum phase was analyzed in participants who reported engaging in such behaviors prior to birth. Of these participants, $81.7 \%$ reported reengagement of sexual intercourse within the first 3 months after the birth. Additionally, 69.6\% of co-parents reported engagement in oral sex and $72.7 \%$ reported having masturbated during this time. A repeated measures multivariate analysis of variance (RM MANOVA) showed an overall trend for a significant difference for engagement in these three sexual activities in the postpartum period, $F(2,128)=3.03, P=0.058$, such that co-parents were more likely to have engaged in intercourse over oral sex or masturbation (all P's < 0.032). Post hoc analyses also showed no significant differences in engagement of oral sex or masturbation postpartum $(P=0.843)$.

Frequencies of resumption across the 3 -month postpartum period for each sexual activity are listed in Table 2. Co-parents showed peak resumption frequencies early in the postpartum period, from 0 to 6 weeks, for activities that did not involve their partner's vaginas and vulvas (i.e., oral sex on the co-parent and masturbation). Resumption of activities involving their female partner's genitals (i.e., intercourse; oral sex on the birth mother) appeared to be consistent throughout the postpartum period with approximately one-third of co-parents reporting resumption at each postpartum time point. An RM MANOVA, using four sexual activities (i.e., intercourse, oral sex on the co-parent, oral sex on the birth mother, and masturbation) was conducted to determine differences in the times to reengagement for each sexual activity. An overall significant difference in time to reengagement was found, $F(3,87)=6.22$, $P=0.002$. Reengagement in masturbation occurred significantly earlier in the postpartum period than intercourse $(P<0.001)$, oral sex on the co-parent $(P<0.048)$, and oral sex on the birth mother $(P<0.001)$. There was a trend for significantly earlier resumption of oral sex on the co-parent over oral sex on the birth mother $(P<0.068)$; however, there were no significant differences in time to reengagement between intercourse and oral sex on either the co-parent or the birth mother. These findings thus suggest a greater focus on solitary sexuality early in the postpartum period, over activities where their partners (i.e., the birth mothers) are involved.

In exploring other potential factors that may influence resumption of sexual activities, birth experience (QMAALD), social support (MSPSS), stress (PSS), body image self-consciousness (BISC), birth trauma, fatigue, and partner's breastfeeding status were considered as covariates, yet this did not change the pattern of activity resumption. Additionally, including co-parents' perceptions of their partner's desire, as well as their ratings for importance of fulfilling their partner's desire, did not alter the overall pattern of activity

Table 2 Time to resumption of sexual activity following childbirth

\begin{tabular}{|c|c|c|c|c|c|c|c|c|}
\hline \multirow[b]{3}{*}{ Activity } & \multicolumn{6}{|c|}{ Time to resumption from birth* } & & \\
\hline & \multicolumn{2}{|c|}{ 0-6 weeks } & \multicolumn{2}{|c|}{$7-12$ weeks } & \multicolumn{2}{|c|}{$>12$ weeks } & \multicolumn{2}{|c|}{ Total reengagemen } \\
\hline & $\mathrm{N}$ & $\%$ & $\mathrm{~N}$ & $\%$ & $\mathrm{~N}$ & $\%$ & $\mathrm{~N}$ & $\%$ \\
\hline Vaginal intercourse & 26 & 34.2 & 27 & 35.5 & 22 & 28.9 & 75 & 100 \\
\hline Oral sex: co-parent receiving & 32 & 58.2 & 10 & 18.2 & 10 & 18.2 & 52 & 95 \\
\hline Oral sex: birth mother receiving & 18 & 32.7 & 17 & 30.9 & 14 & 25.5 & 49 & 89 \\
\hline Masturbation & 48 & 73.8 & 8 & 12.3 & 7 & 10.8 & 63 & 100 \\
\hline
\end{tabular}

*Only co-parents who reported prebirth engagement in each activity are included in each category 
reengagement. This suggests that co-parents' sexual behavior in the postpartum period may be less affected by psychosocial or relational context.

To determine differences in enjoyment among intercourse, receipt of oral sex, and masturbation, a RM MANOVA was conducted using these three sexual activities. A significant overall effect was found for enjoyment, and pairwise comparisons showed that co-parents enjoyed receiving oral sex more so than intercourse or masturbation (all $P$ 's $<0.030)$. There were no significant differences in enjoyment of intercourse and masturbation $(P=0.902)$.

To further characterize co-parents' experience of sexuality, chi-square tests were done to determine who was responsible for initiation of the first sexual activities in the postpartum period. There were no significant differences in initiation by the co-parent or by the birth mother for first postpartum intercourse, $\chi^{2}(1)=0.84, P=0.359$. The first performance of oral sex on the co-parent was initiated more often by the birth mother, $\chi^{2}(1)=10.67, P=0.001$, while performance of oral sex on the birth mother was more often initiated by the co-parent, $\chi^{2}(1)=9.68, P=0.002$.

\section{Effects on Postpartum Sexual Desire}

To investigate influences on co-parents' sexual desire in the postpartum period, this study presented participants with a set list of potentially influencing factors, then analyzed which factors participants selected as most contributing to changes in their sexual desire. Each factor was assigned an overall rank based on the number of times it was selected as the first, second, or third most contributing factor to participants' highest and again for their lowest experience of sexual desire. Additionally, each factor was ranked for the number of times it was selected as the \#1 most contributing factor. Relative ranks of factors contributing to bigh desire are listed in Table 3. Factors related to sexual and intimate feelings in participants and their partners were highly ranked overall, as well as for the \#1 most contributing factor, suggesting that an interest in being sexual is more important for high sexual desire than other psychosocial factors such as social support or stress and physical factors such as vaginal injury to the birth mother or energy level. In contrast, when considering factor ranking for low desire (see Table 4), fatigue and stress were highly ranked overall and were likely to be ranked as a top influence. "Amount of available time" was the third most commonly selected factor overall for contribution to participants' lowest experience of postpartum sexual desire; however, it was ranked eighth in selection for the \#1 most contributing factor. Thus, time availability plays a large part in limiting sexual desire and sexual behavior in the postpartum period, but other factors may be more limiting than the lack of time itself.

Given that participants perceived certain factors as having an impact on their sexuality, analyses focused on whether these factors correlated with SDI scores. A series of Pearson's correlations showed no significant correlations between social support from their partner, friends, or family members and SDI scores (all P's > 0.485). Scores on the PSS, BISC, and participants' energy ratings were not significantly associated with dyadic, solitary, or overall sexual desire (all $P$ 's $>0.054$ ). Though co-parents ranked, "My partner's level of

Table 3 Frequency of factors influencing postpartum point of highest sexual desire by rank

\begin{tabular}{|c|c|c|c|c|c|c|}
\hline \multicolumn{3}{|c|}{ Top three selected } & \multirow[b]{2}{*}{ Item } & \multicolumn{3}{|c|}{ First Selected } \\
\hline Rank & $\mathrm{N}$ & $\%$ & & Rank & $\mathrm{N}$ & $\%$ \\
\hline 1 & 60 & 55.0 & Amount of sexual feelings & 3 & 19 & 17.4 \\
\hline 2 & 56 & 51.3 & My partner's level of interest in being sexual with me & 1 & 25 & 22.9 \\
\hline 3 & 49 & 47.1 & Amount of intimate or close feelings toward my partner & 2 & 24 & 22.0 \\
\hline 4 & 30 & 27.5 & Amount of available time & 5 & 6 & 5.5 \\
\hline 5 & 20 & 18.3 & My hormones & 4 & 7 & 6.4 \\
\hline 6 & 17 & 15.6 & Baby's sleeping habits & 5 & 6 & 5.5 \\
\hline 7 & 16 & 14.7 & Overall degree of vaginal discomfort from birth & 11 & 0 & 0 \\
\hline 8 & 14 & 12.8 & Breastfeeding & 8 & 3 & 2.8 \\
\hline 9 & 11 & 10.1 & Degree of vaginal dryness & 7 & 4 & 3.7 \\
\hline 10 & 8 & 7.3 & Personal level of fatigue & 7 & 4 & 3.7 \\
\hline 10 & 8 & 7.3 & Amount of stress & 9 & 2 & 1.8 \\
\hline 11 & 7 & 6.4 & Amount of social support from others & 9 & 2 & 1.8 \\
\hline 12 & 6 & 5.5 & Degree of vaginal bleeding from birth & 10 & 1 & 0.9 \\
\hline
\end{tabular}


Table 4 Frequency of factors influencing postpartum point of lowest sexual desire by rank

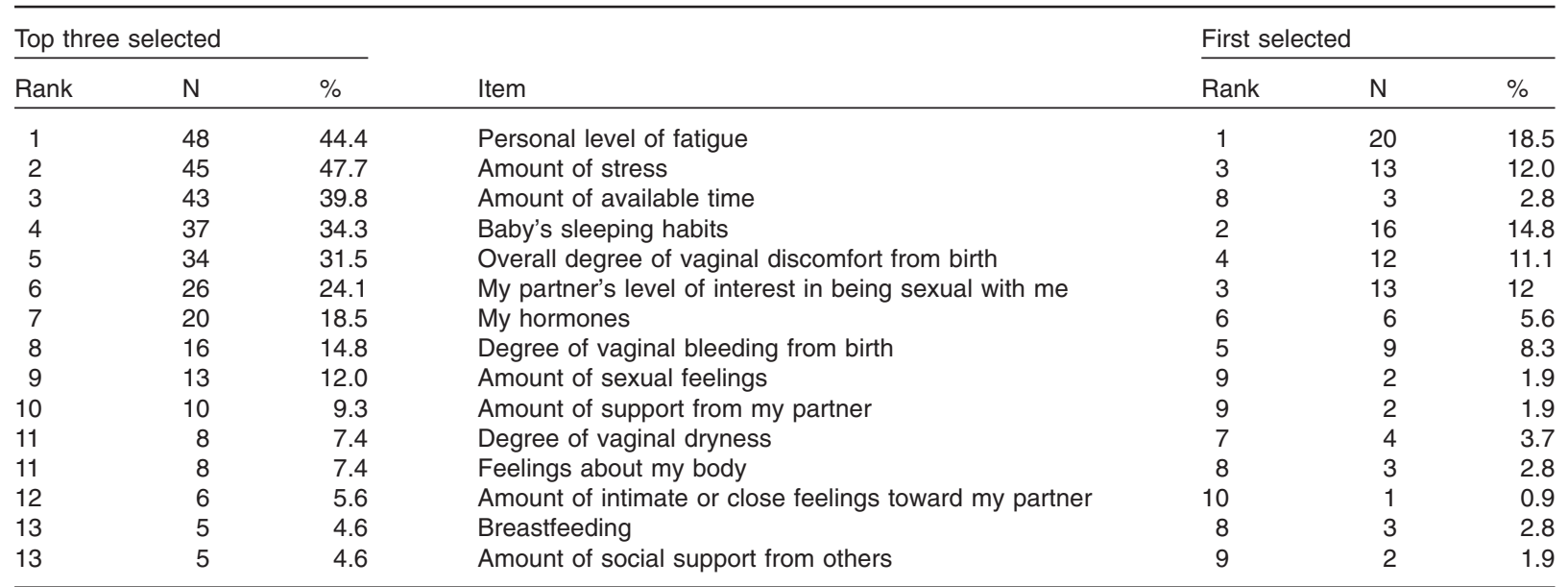

interest in being sexual with me," highly in the factor ranking task, their perceptions of their partner's level of desire were not found to correlate with reports of their own desire, ascertained from the SDI (all P's > 0.101).

Psychosocial factors surrounding the birth were also explored for associations with sexual desire. Pearson's correlations conducted using QMAALD and SDI scores found no significant correlation with SDI scores (all $P$ 's $>0.094$ ). To further investigate the effects of birth experience, a $t$-test was conducted using the presence of vaginal intervention (e.g., episiotomy, sutures, or assisted vaginal extraction) in the participants' partners as the grouping variable. No significant differences in SDI scores were found for participants whose partners have had interventions and those whose partners did not (all $P$ 's $>0.199$ ). Additionally, participants were asked how they felt their presence during the birth influenced their partner's (i.e., the birth mother's) sexual desire toward them. Analyses using Pearson's correlations showed that these ratings were not associated with participants' SDI scores (all P's > 0.597).

Due to the fact that previous literature discusses the negative impact of gender role conformity on men's postpartum experiences, specifically from pressures to be the primary financial provider and feelings of exclusion from infant caretaking, analyses investigated whether these issues would influence co-parents' sexual desire. Results from a $t$-test showed that co-parents who were employed at the time their child was born had significantly higher solitary SDI scores than those who were not employed, $P=0.022$. Additionally, responses to role conformity questions were not correlated with co-parents' scores on the SDI (all P's >0.324), further indicating that conformity to traditional gender roles had less of an influence on experiences of sexuality postpartum than previously expected. Though multiple studies suggest that feelings of jealousy surrounding the mother-infant breastfeeding dyad have negative effects on postpartum sexuality, results from an analysis of variance with groups based on breastfeeding status (i.e., exclusively breastfeeding from breast, breastfeeding from breast and bottlefeeding, exclusively bottlefeeding with breast milk, and exclusively bottlefeeding with formula) showed no significant differences on SDI scores between groups (all $P$ 's $>0.0617)$. These findings thus suggest that degree of co-parent involvement in infant feeding does not contribute to experiences of postpartum sexual desire.

\section{Gender-Specific Experiences During the Postpartum}

As the postpartum experiences of same-sex couples have not been studied, analyses were conducted to explore how gender of co-parents might be implicated in experiences of sexuality in the first 3 months following the birth of their youngest child, though n's were very small for women (which we report for each analysis) such that these analyses are extremely underpowered.

A series of $t$-tests were conducted using co-parent gender/sex as the grouping variable and the psychosocial variables of interest as the test variables. No significant differences between groups were found in reports of birth experience $(\mathrm{QMAALD})\left(\mathrm{n}_{\text {women }}=18\right)$, perceived stress $(\mathrm{PSS})$ $\left(\mathrm{n}_{\text {women }}=17\right)$, body image self-consciousness (BISC) $\left(\mathrm{n}_{\text {women }}=16\right)$, or average level of fatigue 
$\left(\mathrm{n}_{\text {women }}=18\right)($ all $P$ 's $>0.40)$. Interestingly, significant differences were found in co-parents' perceptions of social support such that co-parent women perceived more support from their significant others (MSPSS-SO) and their friends (MSPSSFRI) and had significantly higher overall social support scores (MSPSS-Total) than male co-parents $\left(\mathrm{n}_{\text {women }}=17\right)$ (all P's $\left.<0.035\right)$. Scores based on social support from family members (MSPSS-FAM) did not differ significantly between groups $(P=0.404)$.

To investigate influences of co-parent gender on the reengagement of the three sexual behaviors, an RM MANOVA was conducted using gender/ sex as the between subjects independent variable $\left(\mathrm{n}_{\text {women }}=9\right)$. Though no overall main effect of gender/sex was found, $F(1,62)=0.62, P=0.436$, a trend for an interaction between activity and gender was noted, $F(2,61)=3.13, P=0.051$. Male co-parents were more likely to have engaged in sexual activity involving penetration than female co-parents $(P=0.022)$. There were no significant differences by gender/sex in engagement of either oral sex or masturbation. Due to sample size limitations $\left(\mathrm{n}_{\text {women }}=3\right)$, analyses could not be completed regarding the effects of gender/sex on the order of activity resumption, activity enjoyment, and activity initiation.

To determine gender/sex influences specific to sexual desire, a $t$-test was conducted using gender/ sex as the grouping variable $\left(\mathrm{n}_{\text {women }}=14\right)$. No significant differences between groups were found on dyadic, solitary, or overall SDI scores (all $P$ 's $>0.0426)$. Analysis of partner perception scores (i.e., how co-parents perceived their partner's postpartum desire) also showed no significant differences between groups, thus indicating that co-parents' desire is not influenced by how high or low they perceive their partner's desire to be $\left(\mathrm{n}_{\text {women }}=16\right)$. These findings indicate a separation of sexual desire and sexual behavior, such that levels of sexual desire between male and female co-parents may be similar but sexual behavior may manifest differently between the two groups.

\section{Discussion}

This study examined co-parents' experiences of sexuality in the first 3 months following the birth of their youngest child, which we are defining as the postpartum period. Attention was paid to physical, social, psychological, and relational experiences, and thus a novel perspective on sexuality during the postpartum period was provided using a relational context. This research included a wider range of sexual activities, incorporated psychosocial factor effects on postpartum sexuality, and used a positive sexuality perspective in contrast to more traditional approaches that focus on the detrimental effects of pregnancy and birth.

\section{Understanding Co-Parent Postpartum Experience}

Results from this study suggested that co-parents resumed masturbation earliest in the postpartum period. Interestingly, however, oral sex was deemed more enjoyable, suggesting that enjoyment per se did not drive the early engagement in masturbation. Previous research suggested that this early resumption of masturbation over other partnered sexual activities may be an artifact of fear surrounding the resumption of penetrative intercourse. Yet, results from this study showed that birth experience (QMAALD) and presence of vaginal sutures did not influence the early resumption pattern of masturbation over other partnered sexual activities. Additionally, whether the birth mother had interventions associated with a vaginal delivery (i.e., episiotomy, sutures, or extraction) did not influence co-parents' sexual desire, further implicating factors beyond vaginal healing. It is possible, however, that co-parents' perceptions of the birth or knowledge of their partner's vaginal injury (or lack thereof) did not affect the already largely ingrained script of birth as a traumatic and damaging experience that requires an extended period of abstinence.

In a previous study, a number of psychosocial and relational variables were found to affect birth mothers' sexual desire and activity reengagement postpartum [1]. In contrast, the present study with co-parents did not uncover associations between these factors and desire or reengagement patterns, including no effects of co-parents' perceptions of the birth mother's sexual desire, importance of her sexual fulfillment, perceptions of social support, or personal fatigue. The one factor that was implicated for postpartum desire was the employment status of the co-parent, such that employed co-parents had higher scores on the SDI than co-parents who were not employed during this time. This single link suggests that co-parent desire may be affected by factors beyond the scope of the social/relational context explored by this study. Multiple external factors surrounding employment, including job satisfaction, income level (i.e., concerns regarding the financial burden of raising a child), job support, and parental leave 
policies, may also contribute, though further research is necessary to investigate these potential factors.

\section{Influences of Gender on Postpartum Experience}

This study was the first to explore postpartum sexuality in both co-parent women and men. Our sample sizes were extremely small for women co-parents, and results should be interpreted with caution. We found that only perceptions of social support significantly differed between men and women co-parents, and this may be an artifact of gendered development of social networks. Vaux noted that women's social networks are made up of more expansive, intimate friendships, while men tend to restrict social intimacy to formal relationship, often consisting of only a partner or spouse [26]. Thus, in the postpartum period, co-parent women may be better able to navigate challenges following the birth of their child by reaching out to friends and by being more expressive of their concerns to their partner (i.e., the birth mother). In contrast, male co-parents of birth mothers may feel that they have lost their only source of emotional support as their partner focuses her attention on the baby. Additionally, gender differences in social support navigation might be amplified during times of stress and thus may become more profound during the postpartum period [26,27].

Co-parent men were significantly more likely to have engaged in penetrative sex than co-parent women. Olsson et al. noted the pressures men feel to adhere to masculine stereotypes regarding participation in sexual activity, the absence of which for women co-parents may mean less pressure to reengage in penetrative sex in the postpartum period [11]. Thus, external pressures and adherence to gender-based stereotypes regarding sexual behavior may contribute to the differences noted in this study.

\section{Conceptualizing Postpartum Sexuality}

Results from this study and previous research suggest that postpartum sexuality can be conceptualized as an individualized experience within a partnership, as well as one that occurs in a larger social and relational context. Responses from parous women in our earlier work regarding the rate of engagement in postpartum intercourse and oral sex were similar to those of the co-parents in this study as $82 \%$ of co-parents engaged in intercourse and $70 \%$ of co-parents reported engaging in oral sex during this time. Additionally, both groups ranked "Amount of sexual feelings," "My partner's level of interest in being sexual with me," and "Amount of intimate or close feelings toward my partner" as the top three most frequent influences on high desire. Thus, the present results act as a quasireplication and validation for both studies, in that birth mothers and co-parents, separately surveyed and largely not partnered with each other, independently reported similar postpartum sexual experiences. Other findings, however, showed that postpartum sexual experiences can differ between birth mothers and co-parents despite these similarities. While birth mothers showed highest enjoyment for masturbation [1], co-parents in this study demonstrated highest enjoyment for receiving oral sex, suggesting differences in preferences for solitary and dyadic sexual activity. Additionally, the fact that parous women's sexual desire was found to be correlated with psychosocial and relational variables (e.g., perceptions of a partner's desire, importance of partner's sexual fulfillment, and fatigue), while co-parents' desires were not, suggests a nuanced approach to the study of postpartum sexuality, perhaps with a more individualized focus for co-parents. Further research is thus needed to flesh out the novel influences on co-parents' sexual desire, those which may or may not be independent of their experiences within their relationship.

Though this study provided a much needed focus on the experiences of co-parents during the first 3 months after the birth of their child, it has limitations. One limitation is the use of retrospective report to gain responses from co-parents. Accuracy of participant responses may thus be adversely affected given that considerable and varying time had passed since the postpartum time period. Additionally, the scales used in this study have not been validated for retrospective use or for use in co-parents, which is an important limitation. These scales, however, have been used retrospectively in the previous study specific to women's postpartum sexuality [1], with results that converged with findings in this paper, adding validity to the usefulness of these scales as retrospective measures. This retrospective method allowed for recruitment of a larger and more diverse participant population, particularly by increasing the number of co-parent women eligible for participation. However, shorter periods of recall may be associated with different patterns of evidence; our own sample was relatively small to conduct year-by-year analyses and was not designed to do this, so future research should compare how time since birth may be associated 
with different patterns of recollected experiences (which is interesting for reasons beyond methodological as well).

Though this research attempts to be inclusive of co-parent diversity, these findings were not representative of all co-parents as analyses were limited to co-parents in a sexual relationship during the postpartum period, and only a small number of responses from co-parent women were obtained. Limits from small sample sizes thus restricted broader analyses based on co-parent gender, and findings from the comparisons done in this study should be replicated using larger samples. Additionally, because of the volunteer self-report nature of this research, it is possible that only co-parents with an interest in postpartum sexuality or their own postpartum experiences completed the questionnaire. It is thus possible that the findings from this study do not fully represent postpartum sexuality for all co-parents, suggesting limits to generalizability. Moreover, the ways in which postpartum sexuality might be experienced for co-partners could differ very markedly depending on participants' ages or the ages of their partners as well as other important social location factors. Finally, this study did not include other sexual intimacies including kissing, cuddling, hugging, etc., and thus further exploration of these aspects of co-parent sexuality during the postpartum period is warranted. Despite these limitations, this study offers a contemporary lens to understanding the experiences of co-parents' sexuality during the postpartum period using a population sample that extends beyond heterosexual married men and considers sexual activities beyond penetrative intercourse.

\section{Conclusions}

Exploration into the experiences of co-parents highlights both similarities and key differences in the way sexuality is experienced between members of the postpartum couple. This study bridges these two perspectives to create a broader understanding of the experience of sexuality in the postpartum period, one that conceptualizes co-parent sexuality as unique and not entirely congruent with the experiences of their parous partners. Though further investigation is necessary to consider alternative influences on co-parent desire, this study establishes an enhanced understanding of co-parents' experiences of sexuality within the previously parous women-centered realm that is the postpartum period. This new understanding can aid health professionals in counseling women during this phase postchildbirth, and expand understandings of postpartum sexuality from a biomedical to sociorelational one as well as part of the broader sexual realm.

Corresponding Author: Sari M. van Anders, $\mathrm{PhD}$, Departments of Psychology \& Women's Studies, Program in Neuroscience, Reproductive Science Program, University of Michigan, 530 Church Street, Ann Arbor, MI 48109, USA. Tel: (1) 734-647-6981; Fax: (1) 734-763-7480; E-mail: smva@umich.edu

Conflict of Interest: The authors report no conflicts of interest.

\section{Statement of Authorship}

\section{Category 1}

(a) Conception and Design

Sari M. van Anders; Lauren E. Hipp; Lisa Kane Low

(b) Acquisition of Data

Sari M. van Anders; Lauren E. Hipp

(c) Analysis and Interpretation of Data

Sari M. van Anders; Lauren E. Hipp; Lisa Kane Low

\section{Category 2}

(a) Drafting the Article

Sari M. van Anders; Lauren E. Hipp

(b) Revising It for Intellectual Content Sari M. van Anders; Lauren E. Hipp; Lisa Kane Low

\section{Category 3}

(a) Final Approval of the Completed Article Sari M. van Anders; Lauren E. Hipp; Lisa Kane Low

\section{References}

1 Hipp LE, Kane Low L, van Anders SM. Exploring women's postpartum sexuality: Social, psychological, relational, and birth-related contextual factors. J Sex Med 2012;9:2330-41.

2 von Sydow K. Sexuality during pregnancy and after childbirth: A metacontent analysis of 59 studies. J Psychosom Res 1999;47:27-49.

3 Harriman LC. Personal and marital changes accompanying parenthood. Fam Relat 1983;32:387-94.

4 Landis JT, Poffenberger T, Poffenberger S. The effects of first pregnancy upon the sexual adjustment of 212 couples. Am Sociol Rev 1950;15:766-72.

5 Fischman SH, Rankin EA, Soeken KL, Lenz ER. Changes in sexual relationships in postpartum couples. J Obstet Gynecol Neonatal Nurs 1986;15:58-63.

6 Gordon IB, Carty E. Sexual adjustment of postpartum couples. Can Fam Physician 1978;24:1191-8.

7 Condon JT, Boyce P, Corkindale CJ. The first-time fathers study: A prospective study of the mental health and wellbeing 
of men during the transition to parenthood. Aust N Z J Psychiatry 2004;38:56-64.

8 Haugen EN, Schmutzer PA, Wenzel A. Sexuality and the partner relationship during pregnancy and the postpartum period. In: Harvey JH, Wenzel A, Sprecher S, eds. The handbook of sexuality in close relationships. Mahwah, NJ: Lawrence Erlbaum Associates; 2004:411-35.

9 Hyde JS, DeLamater JD, Plant EA, Byrd JM. Sexuality during pregnancy and the year postpartum. J Sex Res 1996;33:143-51.

10 Byrd JE, Hyde JS, DeLamater JD, Plant EA. Sexuality during pregnancy and the year postpartum. J Fam Pract 1998;47: $305-8$.

11 Olsson A, Robertson E, Bjrklund A, Nissen E. Fatherhood in focus, sexual activity can wait: New fathers' experience about sexual life after childbirth. Scand J Caring Sci 2010;15:716-25.

12 Cowan CP, Cowan PA, Heming G, Garrett E, Coysh WS, Curtis-Boles H, Boles AJ III. Transitions to parenthood: His, hers, and theirs. J Fam Issues 1985;6:451-81.

13 Ahlborg T, Strandmark M. The baby was the focus of attention-First-time parents' experiences of their intimate relationship. Scand J Caring Sci 2001;15:318-25.

14 Ahlborg T, Strandmark M. Factors influencing the quality of intimate relationships six months after delivery- first-time parents' own views and coping strategies. J Psychosom Obstet Gynaecol 2006;27:163-72.

15 Fagerskiold A. A change in life as experienced by first-time fathers. Scand J Caring Sci 2008;22:64-71.

16 Gungor S, Baser I, Ceyhan T, Karasahin E, Kilic S. Does mode of delivery affect sexual functioning of the man partner? J Sex Med 2008;5:155-63.
17 Safarinejad MR, Kolahi AA, Hosseini L. The effect of the mode of delivery on the quality of life, sexual function, and sexual satisfaction in primiparous women and their husbands. J Sex Med 2009;6:1645-67.

18 Hames CT. Sexual needs and interests of postpartum couples. J Obstet Gynecol Neonatal Nurs 1980;9:313-5.

19 Jordan PL, Wall VR. Supporting the father when an infant is breastfed. J Hum Lact 1993;9:31-4.

20 Buhrmester M, Kwang T, Gosling SD. Amazon's Mechanical Turk: A new source of inexpensive, yet high-quality, data? Perspect Psychol Sci 2011;6:3-5.

21 Marut JS, Mercer RT. Comparison of primiparas' perceptions of vaginal and cesarean births. Nurs Res 1979;28: 260-6.

22 Zimet G, Dahlem N, Zimet S, Farley G. The multidimensional scale of perceived social support. J Pers Assess 1988;52:30-41.

23 Cohen S, Kamarck T, Mermelstein R. A global measure of perceived stress. J Health Soc Behav 1983;24:385-96.

24 Wiederman M. Women's body image self-consciousness during physical intimacy with a partner. J Sex Res 2000;37: $60-8$.

25 Spector IP, Carey MP, Steinberg L. The sexual desire inventory: Development, factor structure, and evidence of reliability. J Sex Marital Ther 1996;22:175-90.

26 Vaux A. Variations in social support associated with gender, ethnicity, and age. J Soc Issues 1985;41:89-110.

27 Caldwell RA, Bloom BL. Social support: Its structure and impact on marital disruption. Am J Community Psychol 1982;10:647-67. 